\title{
Semiconductors and Dirichlet-to-Neumann maps
}

\author{
ANTONIO LEITÃO \\ Department of Mathematics, Federal University of St. Catarina \\ 88040-900 Florianopolis, Brazil \\ E-mail: aleitao@mtm.ufsc.br
}

\begin{abstract}
We investigate the problem of identifying discontinuous doping profiles in semiconductor devices from data obtained by the stationary voltage-current (VC) map. The related inverse problem correspond to the inverse problem for the Dirichlet-to-Neumann (DN) map with partial data.
\end{abstract}

Mathematical subject classification: $35 \mathrm{R} 30,82 \mathrm{D} 37,35 \mathrm{Q} 60$.

Key words: semiconductors, inverse doping, bipolar model.

\section{Introduction}

In this paper we investigate the problem of identifying discontinuous doping profiles in semiconductor devices from data obtained by the VC map for the linearized stationary bipolar model (close to equilibrium). Two different methods of data acquisition are considered:

1) Current flow measurements through a contact;

2) Pointwise measurements of the current density.

The related inverse problems correspond to the inverse problem for the DN map with partial data.

We propose a framework to handle the inverse problems and analyze relevant properties of the parameter-to-output maps. Moreover, we present a numerical experiment for the case of pointwise measurements of the current density. 
The paper is outlined as follows: In Section 2 we present the transient end stationary drift diffusion equations. In the Section 3 we introduce the VC map and derive the underlying model for the analysis presented in this paper, namely the linearized stationary bipolar case (close to equilibrium). Two inverse problems corresponding to different data acquisition procedures are introduced in Section 4. Some regularity properties of the related parameter-to-output maps are verified in this section. In Section 5 we present some numerical results for a level set type iterative method and pointwise measurements of the current density. This experiment indicate that a single measurement may suffices to identify the doping profile.

\section{Drift diffusion equations}

\section{The transient model}

The basic semiconductor device equations consist of the Poisson equation (1a), the continuity equations for electrons (1b) and holes (1c), and the current relations for electrons (1d) and holes (1e).

$$
\begin{array}{ll}
\operatorname{div}(\epsilon \nabla V)=q(n-p-C) & \text { in } \Omega \times(0, T) \\
\operatorname{div} J_{n}=q\left(\partial_{t} n+R\right) & \text { in } \Omega \times(0, T) \\
\operatorname{div} J_{p}=q\left(-\partial_{t} p-R\right) & \text { in } \Omega \times(0, T) \\
J_{n}=q\left(D_{n} \nabla n-\mu_{n} n \nabla V\right) & \text { in } \Omega \times(0, T) \\
J_{p}=q\left(-D_{p} \nabla p-\mu_{p} p \nabla V\right) & \text { in } \Omega \times(0, T) .
\end{array}
$$

This system is defined in $\Omega \times(0, T)$, where $\Omega \subset \mathbb{R}^{d}(d=1,2,3)$ is a domain representing the semiconductor device. Here $V$ denotes the electrostatic potential $(-\nabla V$ is the electric field, $E=|\nabla V|)), n$ and $p$ are the concentration of free carriers of negative charge (electrons) and positive charge (holes) respectively and $J_{n}$ and $J_{p}$ are the densities of the electron and the hole current respectively. $D_{n}$ and $D_{p}$ are the diffusion coefficients for electrons and holes respectively. $\mu_{n}$ and $\mu_{p}$ denote the mobilities of electrons and holes respectively. The positive constants $\epsilon$ and $q$ denote the permittivity coefficient (for silicon) and the elementary charge (see Appendix). 
The function $R$ has the form $R=\mathcal{R}(n, p, x)\left(n p-n_{i}^{2}\right)$ and denotes the recombination-generation rate ( $n_{i}$ is the intrinsic carrier density). The bandgap is relatively large for semiconductors (gap between valence and conduction band), and a significant amount of energy is necessary to transfer electrons from the valence and to the conduction band. This process is called generation of electron-hole pairs. On the other hand, the reverse process corresponds to the transfer of a conduction electron into the lower energetic valence band. This process is called recombination of electron-hole pairs. In our model these phenomena are described by the recombination-generation rate $R$. Frequently adopted in the literature are the Shockley $\operatorname{Read} \operatorname{Hall} \operatorname{model}\left(\mathcal{R}_{S R H}\right)$ and the Auger model $\left(\mathcal{R}_{A U}\right)$, defined by

$$
\begin{aligned}
\mathcal{R}_{S R H} & :=\left[\tau_{p}\left(n+n_{i}\right)+\tau_{p}\left(p+n_{i}\right)\right]^{-1}, \\
\mathcal{R}_{A U} & :=C_{n} n+C_{p} p
\end{aligned}
$$

where $C_{n}, C_{p}, \tau_{n}$ and $\tau_{p}$ are positive constants (see Appendix).

The function $C(x)$ models a preconcentration of ions in the crystal, so $C(x)=$ $C_{+}(x)-C_{-}(x)$ holds, where $C_{+}$and $C_{-}$are concentrations of negative and positive ions respectively. In those subregions of $\Omega$ in which the preconcentration of negative ions predominate (P-regions), we have $C(x)<0$. Analogously, we define the $\mathrm{N}$-regions, where $C(x)>0$ holds. The boundaries between the P-regions and N-regions (where $C$ change sign) are called pn-junctions.

In the sequel we turn our attention to the boundary conditions. We assume the boundary $\partial \Omega$ of $\Omega$ to be divided into two nonempty disjoint parts: $\partial \Omega=$ $\overline{\partial \Omega_{N}} \cup \overline{\partial \Omega_{D}}$. The Dirichlet part of the boundary $\partial \Omega_{D}$ models the Ohmic contacts, where the potential $V$ as well as the concentrations $n$ and $p$ are prescribed. The Neumann part $\partial \Omega_{N}$ of the boundary corresponds to insulating surfaces, thus a zero current flow and a zero electric field in the normal direction are prescribed. The Neumann boundary conditions for system (1a)-(1e) read:

$$
\frac{\partial V}{\partial v}(x, t)=\frac{\partial n}{\partial v}(x, t)=\frac{\partial p}{\partial v}(x, t)=0, \partial \Omega_{N} \times[0, T] .
$$

Moreover, at $\partial \Omega_{D} \times[0, T]$, the following Dirichlet boundary conditions are 
imposed:

$$
\begin{aligned}
& V(x, t)=V_{D}(x, t)=U(x, t)+V_{b i}(x)=U(x, t)+U_{T} \ln \left(\frac{n_{D}(x)}{n_{i}}\right) \\
& n(x, t)=n_{D}(x)=\frac{1}{2}\left(C(x)+\sqrt{C(x)^{2}+4 n_{i}^{2}}\right) \\
& p(x, t)=p_{D}(x)=\frac{1}{2}\left(-C(x)+\sqrt{C(x)^{2}+4 n_{i}^{2}}\right) .
\end{aligned}
$$

Here the function $U(x, t)$ denotes the applied potential, the constant $U_{T}$ represents the thermal voltage, and $V_{b i}$ is given logarithmic function [4]. We shall consider the simple situation $\partial \Omega_{D}=\Gamma_{0} \cup \Gamma_{1}$, which occurs, e.g., in a diode. The disjoint boundary parts $\Gamma_{i}, i=0,1$, correspond to distinct contacts. Differences in $U(x)$ between different segments of $\partial \Omega_{D}$ correspond to the applied bias between these two contacts. Moreover, the initial conditions $n(x, 0) \geq 0$, $p(x, 0) \geq 0$ have to be imposed.

\section{The stationary model}

In this paragraph we turn our attention to the stationary drift diffusion equations. We disconsider the thermal effects and assume further $\partial n / \partial t=\partial n / \partial t=0$. Thus, the stationary drift diffusion model is derived from (1a)-(1e) in a straightforward way. Next, motivated by the Einstein relations $D_{n}=U_{T} \mu_{n}$ and $D_{p}=U_{T} \mu_{p}$ (a standard assumption about the mobilities and diffusion coefficients), one introduces the so-called Slotboom variables $u$ and $v$, which are related to the original $n$ and $p$ variables by the formula:

$$
n(x)=n_{i} \exp \left(\frac{V(x)}{U_{T}}\right) u(x), \quad p(x)=n_{i} \exp \left(\frac{-V(x)}{U_{T}}\right) v(x) .
$$

For convenience, we rescale the potential and the mobilities, i.e.

$$
V(x) \leftarrow \frac{V(x)}{U_{T}}, \mu_{n} \leftarrow q U_{T} \mu_{n}, \mu_{p} \leftarrow q U_{T} \mu_{p} .
$$

It is obvious to check that the current relations now read $J_{n}=\mu_{n} n_{i} e^{V} \nabla u$, $J_{p}=-\mu_{p} n_{i} e^{-V} \nabla v$. 
Next we write the stationary drift diffusion equations in terms of $(V, u, v)$

$$
\begin{array}{ll}
\lambda^{2} \Delta V=\delta^{2}\left(e^{V} u-e^{-V} v\right)-C(x) & \text { in } \Omega \\
\operatorname{div} J_{n}=\delta^{4} Q(V, u, v, x)(u v-1) & \text { in } \Omega \\
\operatorname{div} J_{p}=-\delta^{4} Q(V, u, v, x)(u v-1) & \text { in } \Omega \\
V=V_{D}=U+V_{\mathrm{bi}} & \text { on } \partial \Omega_{D} \\
u=u_{D}=e^{-U} & \text { on } \partial \Omega_{D} \\
v=v_{D}=e^{U} & \text { on } \partial \Omega_{D} \\
\nabla V \cdot v=J_{n} \cdot v=J_{p} \cdot v=0 & \text { on } \partial \Omega_{N},
\end{array}
$$

where $\lambda^{2}:=\epsilon /\left(q U_{T}\right)$ is the Debye length of the device, $\delta^{2}:=n_{i}$ and the function $Q$ is defined implicitly by the relation $Q(V, u, v, x)=\mathcal{R}(n, p, x){ }^{1}$

One should notice that, due to the thermal equilibrium assumption, it follows $n p=n_{i}^{2}$, and the assumption of vanishing space charge density gives $n-p-C=$ 0 , for $x \in \partial \Omega_{D}$. This fact motivates the boundary conditions on the Dirichlet part of the boundary.

It is worth mentioning that, in a realistic model, the mobilities $\mu_{n}$ and $\mu_{p}$ usually depend on the electric field strength $|\nabla V|$. In what follows, we assume that $\mu_{n}$ and $\mu_{p}$ are positive constants. This assumption simplifies the subsequent analysis, allowing us to concentrate on the inverse doping problems. As a matter of fact, this dependence could be incorporated in the model without changing the results described in the sequel.

Existence and uniqueness of solutions for system (5) can only be guaranteed for small applied voltages. Therefore, it is reasonable to consider, instead of this system, its linearized version around the equilibrium point $U \equiv 0$. We shall return to this point in the next section, where the VC map is introduced.

\section{A simplified model}

In the sequel we make some simplifying assumptions on the stationary drift diffusion equations introduced in Section 2 and derive a special case which will serve as underlying model for the inverse problem investigated in Section 4.

\footnotetext{
${ }^{1}$ Notice the applied potential has also to be rescaled: $U(x) \leftarrow U(x) / U_{T}$.
} 


\section{The linearized stationary drift diffusion equations (close to equilibrium)}

We begin this paragraph by introducing the thermal equilibrium assumption for the stationary drift diffusion equations. This is a previous step to derive a linearized system of stationary drift diffusion equations (close to equilibrium).

The thermal equilibrium assumption refers to the condition in which the semiconductor is not subject to external excitations, except for a uniform temperature, i.e. no voltages or electric fields are applied. It is worth noticing that, under the thermal equilibrium assumption, all externally applied potentials to the semiconductor contacts are zero (i.e. $U(x)=0$ ). Moreover, the thermal generation is perfectly balanced by recombination (i.e. $\mathcal{R}=0$ ).

If the applied voltage satisfies $U=0$, one immediately sees that the solution of system $(5 \mathrm{a})-(5 \mathrm{~g})$ simplifies to $(V, u, v)=\left(V^{0}, 1,1\right)$, where $V^{0}$ solves

$$
\begin{array}{ll}
\lambda^{2} \Delta V^{0}=e^{V^{0}}-e^{-V^{0}}-C(x) & \text { in } \Omega \\
V^{0}=V_{\mathrm{bi}}(x) & \text { on } \partial \Omega_{D} \\
\nabla V^{0} \cdot v=0 & \text { on } \partial \Omega_{N} .
\end{array}
$$

In the bipolar model discussed below we shall be interested in the linearized drift diffusion system at the equilibrium. Keeping this in mind, we compute the Gateaux derivative of the solution of system (5a)-(5g) with respect to the voltage $U$ at the point $U \equiv 0$ in the direction $h$. This directional derivative is given by the solution $(\hat{V}, \hat{u}, \hat{v})$ of

$$
\begin{array}{ll}
\lambda^{2} \Delta \hat{V}=e^{V^{0}} \hat{u}+e^{-V^{0}} \hat{v}+\left(e^{V^{0}}+e^{-V^{0}}\right) \hat{V} & \text { in } \Omega \\
\operatorname{div}\left(\mu_{n} e^{V^{0}} \nabla \hat{u}\right)=Q_{0}\left(V^{0}, x\right)(\hat{u}+\hat{v}) & \text { in } \Omega \\
\operatorname{div}\left(\mu_{p} e^{-V^{0}} \nabla \hat{v}\right)=Q_{0}\left(V^{0}, x\right)(\hat{u}+\hat{v}) & \text { in } \Omega \\
\hat{V}=h & \text { on } \partial \Omega_{D} \\
\hat{u}=-h & \text { on } \partial \Omega_{D} \\
\hat{v}=h & \text { on } \partial \Omega_{D} \\
\nabla V^{0} \cdot v=\nabla \hat{u} \cdot v=\nabla \hat{v} \cdot v=0 & \text { on } \partial \Omega_{N},
\end{array}
$$

where the function $Q_{0}$ satisfies $Q_{0}\left(V^{0}, x\right)=Q\left(V^{0}, 1,1, x\right)$. 


\section{Linearized stationary bipolar case (close to equilibrium)}

In this paragraph we present a special case, which plays a key rule in the modeling inverse doping problems related to current flow measurements.

The following discussion is motivated by the stationary VC map

$$
\begin{aligned}
\Sigma_{C}: H^{3 / 2}\left(\partial \Omega_{D}\right) & \rightarrow \mathbb{R} . \\
U & \mapsto \int_{\Gamma_{1}}\left(J_{n}+J_{p}\right) \cdot v d s
\end{aligned}
$$

Here $(V, u, v)$ is the solution of system (5) for an applied voltage $U$. This operator models practical experiments where voltage-current data are available, i.e. measurements of the averaged outflow current density on $\Gamma_{1} \subset \partial \Omega_{D}$.

The linearized stationary bipolar case (close to equilibrium) corresponds to the model obtained from the drift diffusion equations (5) by linearizing the VC map at $U \equiv 0$. This simplification is motivated by the fact that, due to hysteresis effects for large applied voltage, the VC map can only be defined as a single-valued function in a neighborhood of $U=0$. Moreover, the following simplifying assumptions are also taken into account:

A1) The electron mobility $\mu_{n}$ and hole mobility $\mu_{p}$ are constant;

A2) No recombination-generation rate is present, i.e. $\mathcal{R}=0$ (or $Q_{0}=0$ ).

An immediate consequence of our assumptions is the fact that the Poisson equation and the continuity equations decouple. Indeed, from (7) we see that the Gateaux derivative of the VC map $\Sigma_{C}$ at the point $U=0$ in the direction $h \in H^{3 / 2}\left(\partial \Omega_{D}\right)$ is given by the expression

$$
\Sigma_{C}^{\prime}(0) h=\int_{\Gamma_{1}}\left(\mu_{n} e^{V_{b i}} \hat{u}_{v}-\mu_{p} e^{-V_{b i}} \hat{v}_{v}\right) d s,
$$

where $(\hat{u}, \hat{v})$ solve

$$
\begin{array}{ll}
\operatorname{div}\left(\mu_{n} e^{V^{0}} \nabla \hat{u}\right)=0 & \text { in } \Omega \\
\operatorname{div}\left(\mu_{p} e^{-V^{0}} \nabla \hat{v}\right)=0 & \text { in } \Omega \\
\hat{u}=-h & \text { on } \partial \Omega_{D} \\
\hat{v}=h & \text { on } \partial \Omega_{D} \\
\nabla \hat{u} \cdot v=\nabla \hat{v} \cdot v=0 & \text { on } \partial \Omega_{N}
\end{array}
$$


and $V^{0}$ is the solution of the equilibrium problem (6); see Lemma 1.

Notice that the solution of the Poisson equation can be computed a priori, since it does not depend on $h$. The application $\Sigma_{C}^{\prime}(0)$ maps the Dirichlet data for $(\hat{u}, \hat{v})$ to a weighted sum of their Neumann data and can be compared with the DN map in the Electrical Impedance Tomography (EIT).

\section{Inverse Problems for Semiconductors}

We begin this section verifying that the stationary $\mathrm{VC}$ map $\Sigma_{C}$, introduced in Section 3, is well defined in a suitable neighborhood of $U=0$.

Lemma 1 [[5], Proposition 3.1]. In (5), for each applied voltage $U \in B_{r}(0)$ $\subset H^{3 / 2}\left(\partial \Omega_{D}\right)$ with $r>0$ sufficiently small, the current $J \cdot v \in H^{1 / 2}\left(\Gamma_{1}\right)$ is uniquely defined. Furthermore, $\Sigma_{C}: H^{3 / 2}\left(\partial \Omega_{D}\right) \rightarrow H^{1 / 2}\left(\Gamma_{1}\right)$ is continuous and continuously differentiable in $B_{r}(0)$. Moreover, it's derivative in direction $h \in H^{3 / 2}\left(\partial \Omega_{D}\right)$ is given by the operator $\Sigma_{C}^{\prime}(0)$ defined in (8).

As a matter of fact, we can actually prove that, since $(\hat{u}, \hat{v})$ in (9) depend continuously (in $\left.H^{2}(\Omega)^{2}\right)$ on the boundary data $U \in H^{3 / 2}\left(\partial \Omega_{D}\right)$, it follows from the boundedness and compactness of the trace operator $\gamma: H^{2}(\Omega) \rightarrow$ $H^{1 / 2}\left(\Gamma_{1}\right)$ that $\Sigma_{C}^{\prime}(0)$ is a bounded and compact operator.

Lemma 1 establishes a basic property to consider the inverse problem of reconstructing the doping profile $C$ from the VC map. In the sequel we shall consider two possible inverse problems for this map.

\section{Current flow measurements through a contact}

In this first inverse problem we assume that, for each $C$, the output is given by $\Sigma_{C}^{\prime}(0) U_{j}$ for some $U_{j}$. A realistic experiment corresponds to measure, for given $\left\{U_{j}\right\}_{j=1}^{N}$, with $\left\|U_{j}\right\|$ small, the outputs

$$
\left\{\Sigma_{C}^{\prime}(0) U_{j} \mid j=1, \cdots, N\right\}
$$

(recall that $\left.\Sigma_{C}(0)=\left(V^{0}, 1,1\right)\right)$. In practice, the functions $U_{j}$ are chosen to be piecewise constant on the contact $\Gamma_{1}$ and to vanish on $\Gamma_{0}$. From the definition of 
$\Sigma_{C}^{\prime}(0)$ we derive the following abstract formulation of the inverse doping profile problem for the VC map:

$$
F(C)=Y,
$$

where

1) $\left\{U_{j}\right\}_{j=1}^{N} \subset H^{3 / 2}\left(\partial \Omega_{D}\right)$ are fixed voltage profiles satisfying $\left.U_{j}\right|_{\Gamma_{1}}=0$;

2) Parameter: $C=C(x) \in L^{2}(\Omega)=: \chi$;

3) Output: $Y=\left\{\Sigma_{C}^{\prime}(0) U_{j}\right\}_{j=1}^{N} \in \mathbb{R}^{N}=: \mathfrak{y}$;

4) Parameter-to-output map: $F: \mathcal{X} \rightarrow \mathcal{Y}$.

The domain of definition of the operator $F$ is

$$
D(F):=\left\{C \in L^{\infty}(\Omega) ; C_{m} \leq C(x) \leq C_{M} \text {, a.e. in } \Omega\right\},
$$

where $C_{m}$ and $C_{M}$ are suitable positive constants.

The inverse problem described above corresponds to the problem of identifying the doping profile $C$ from the linearized stationary VC map at $U=0$ (see bipolar case in Section 3).

The non-linear parameter-to-output operator $F$ is well defined and Fréchet differentiable in its domain of definition $D(F)$. This assertion follows from standard regularity results in PDE theory (see, e.g., [4], Propositions 2.2 and 2.3).

It is worth noticing that the solution of the Poisson equation can be computed a priori. The remaining problem (coupled system (9) for $(\hat{u}, \hat{v})$ ) is quite similar to the problem of EIT. In this inverse problem the aim is to identify the conductivity $q=q(x)$ in the equation:

$$
-\operatorname{div}(q \nabla u)=f \text { in } \Omega,
$$

from measurements of the Dirichlet-to-Neumann map, which maps the applied voltage $\left.u\right|_{\partial \Omega}$ to the electrical flux $\left.q u_{v}\right|_{\partial \Omega}$. The application $\Sigma_{C}^{\prime}(0)$ maps the Dirichlet data for $\hat{u}$ and $\hat{v}$ to the weighted sum of their Neumann data. It can be seen as the counterpart of electrical impedance tomography for common conducting materials. 


\section{Pointwise measurements of the current density}

In the sequel, we investigate a different inverse problem related to the VC map. Differently from the previous paragraph, we shall assume that the VC operator maps the Dirichlet data for $\hat{u}$ and $\hat{v}$ in (9) to the sum of their Neumann data, i.e.

$$
\begin{aligned}
\Sigma_{C}: H^{3 / 2}\left(\partial \Omega_{D}\right) & \rightarrow H^{1 / 2}\left(\Gamma_{1}\right) \\
U & \left.\mapsto\left(J_{n}+J_{p}\right) \cdot v\right|_{\Gamma_{1}}
\end{aligned}
$$

where functions $V, \hat{u}, \hat{v}, J_{n}, J_{p}$ and $U$ have the same meaning as in Section 3. It is immediate to observe that the Gateaux derivative of the VC map $\Sigma_{C}$ at the point $U=0$ in the direction $h \in H^{3 / 2}\left(\partial \Omega_{D}\right)$ is given by

$$
\Sigma_{C}^{\prime}(0) h=\left.\left(\mu_{n} e^{V_{b i}} \hat{u}_{v}-\mu_{p} e^{-V_{b i}} \hat{v}_{v}\right)\right|_{\Gamma_{1}},
$$

where $(\hat{u}, \hat{v})$ solve system (9). Notice that, for each applied voltage $U$, the VC map associates a scalar valued function defined on $\Gamma_{1}$. In this case, the outputs $\Sigma_{C}^{\prime}(0) U_{j}$ are in a data space which is larger than in the case of current flow measurements.

Again we can derive an abstract formulation of type (10) for the inverse doping profile problem for the linearized stationary VC map with pointwise measurements of the current density. The only difference to the framework described in the previous paragraph concerns the definition of the Hilbert space $Y$, which is now defined by:

3') Output: $Y=\left\{\Sigma_{C}^{\prime}(0) U_{j}\right\}_{j=1}^{N} \in L^{2}\left(\Gamma_{1}\right)^{N}=: \mathfrak{y}$;

The domain of definition of the operator $F$, remains unaltered.

It is immediate to observe that the model concerning current flow measurements carries less information about the unknown parameter than the model related to pointwise measurements does. In so far, the inverse problem related with the first measurement type is harder to solve.

\section{A numerical experiment}

In this section we apply numerical methods to solve an inverse doping profile problem related to the VC map. We consider the stationary linearized bipolar model with pointwise measurements of the current density. 
In the sequel we consider the bipolar model introduced in Section 3. It follows from the assumption $Q=0$ that the Poisson equation (6a) and the continuity equations (9a), (9b) decouple. The inverse doping profile problem corresponds to the identification of $C=C(x)$ from pointwise measurements of the total current density $J$ at the contact $\Gamma_{1}$, namely

$$
\left.J\right|_{\Gamma_{1}}=\left.\left(J_{n}+J_{p}\right)\right|_{\Gamma_{1}}=\left.\left(\mu_{n} e^{V_{\mathrm{bi}}} \hat{u}_{\nu}-\mu_{p} e^{-V_{\mathrm{bi}}} \hat{v}_{\nu}\right)\right|_{\Gamma_{1}}
$$

(compare with the Gateaux derivative of the VC map $\Sigma_{C}$ at the point $U=0$ in (8)). Here $\left(V^{0}, \hat{u}, \hat{v}\right)$ solve, for each applied voltage $U$, the system (6), (9), with $h$ substituted by $U$.

Notice that we can split the inverse problem in two parts: First we define the function $\gamma(x):=e^{V^{0}(x)}, x \in \Omega$, and solve the parameter identification problem

$$
\begin{array}{llll}
\operatorname{div}\left(\mu_{n} \gamma \nabla \hat{u}\right)=0 & \text { in } \Omega & \operatorname{div}\left(\mu_{p} \gamma^{-1} \nabla \hat{v}\right)=0 & \text { in } \Omega \\
\hat{u}=-U(x) & \text { on } \partial \Omega_{D} & \hat{v}=U(x) & \text { on } \partial \Omega_{D} \\
\nabla \hat{u} \cdot v=0 & \text { on } \partial \Omega_{N} & \nabla \hat{v} \cdot v=0 & \text { on } \partial \Omega_{N}
\end{array}
$$

for $\gamma$, from measurements of $\left.\left(\mu_{n} \gamma \hat{u}_{v}-\mu_{p} \gamma^{-1} \hat{v}_{v}\right)\right|_{\Gamma_{1}}$. The second step consists in the determination of $C$ in

$$
C(x)=\gamma(x)-\gamma^{-1}(x)-\lambda^{2} \Delta(\ln \gamma(x)), x \in \Omega .
$$

Since the evaluation of $C$ from $\gamma$ can be explicitely performed in a stable way, we shall focus on the problem of identifying the function parameter $\gamma$ in (12).

Summarizing, the inverse doping profile problem in the linearized bipolar model for pointwise measurements of the current density reduces to the identification of the parameter $\gamma$ in (12) from measurements of the DN map

$$
\begin{aligned}
\Lambda_{\gamma}: H^{3 / 2}\left(\partial \Omega_{D}\right) & \rightarrow H^{1 / 2}\left(\Gamma_{1}\right) \\
U & \left.\mapsto\left(\mu_{n} \gamma \hat{u}_{v}-\mu_{p} \gamma^{-1} \hat{v}_{v}\right)\right|_{\Gamma_{1}}
\end{aligned}
$$

If we take into account the restrictions imposed by the practical experiments described in Section 4, it follows:

i) The voltage profiles $U \in H^{3 / 2}\left(\partial \Omega_{D}\right)$ must satisfy $\left.U\right|_{\Gamma_{1}}=0$; 
ii) The identification of $\gamma$ has to be performed from a finite number of measurements, i.e. from the data

$$
\left\{\left(U_{j}, \Lambda_{\gamma}\left(U_{j}\right)\right)\right\}_{j=1}^{N} \in\left[H^{3 / 2}\left(\Gamma_{0}\right) \times H^{1 / 2}\left(\Gamma_{1}\right)\right]^{N} .
$$

For this experiment concerning pointwise measurements of the current density, we assume that only one measurement is available, i.e. $N=1$ in (13). What concerns the numerical implementation, we applied an iterative method of level set type to solve the identification problem for $\gamma$ in (12) (see $[19,15,20])$. The domain $\Omega \subset \mathbb{R}^{2}$ is the unit square, and the boundary parts are defined as follows

$$
\begin{gathered}
\Gamma_{1}:=\{(x, 1) ; x \in(0,1)\}, \Gamma_{0}:=\{(x, 0) ; x \in(0,1)\}, \\
\partial \Omega_{N}:=\{(0, y) ; y \in(0,1)\} \cup\{(1, y) ; y \in(0,1)\} .
\end{gathered}
$$

The fixed input $U$, is chosen to be a piecewise constant function supported in $\Gamma_{0}$

$$
U(x):= \begin{cases}1, & |x-0.5| \leq h \\ 0, & \text { else }\end{cases}
$$

The doping profile to be reconstructed is shown in Figure 1(a). In Figure 1(b) the voltage source $U$ (applied at $\Gamma_{0}$ ) and the corresponding solution $\hat{u}$ of (12) are shown. In these pictures, as well as in the forthcoming ones, $\Gamma_{1}$ is the lower left edge and $\Gamma_{0}$ is the top right edge (the origin corresponds to the upper right corner).

In Figure 2 we present a numerical experiment for the bipolar model with pointwise measurements of the current density. Here exact data is used for the reconstruction of the p-n junction in Figure 1(a). The pictures show plots of the iteration error after 1, 10 and 100 steps of the level set method respectively.

What concerns the quality of the reconstruction of the P-N junction, the level set approach considered in this paper brings much better results than the LandweberKaczmarz approach implemented in [4]. A possible explanation for the different performance of these methods is the fact that the Landweber-Kaczmarz approach does not take into account the assumption that the coefficient $\gamma$ in (12) for such application is a piecewise constant function. The Landweber-Kaczmarz method tries to identify a real function defined on $\Omega$, which is a much more complicated 

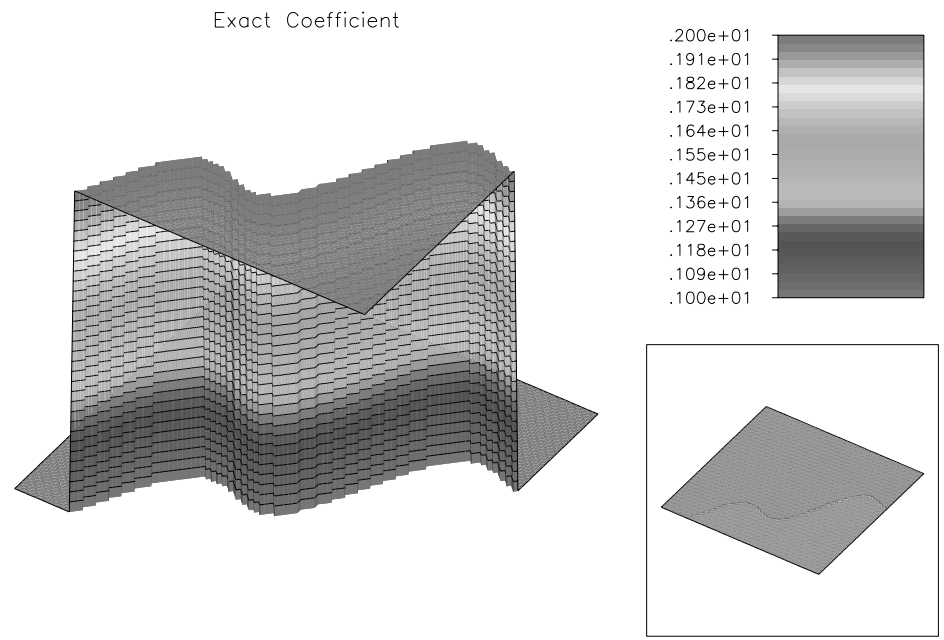

(a)
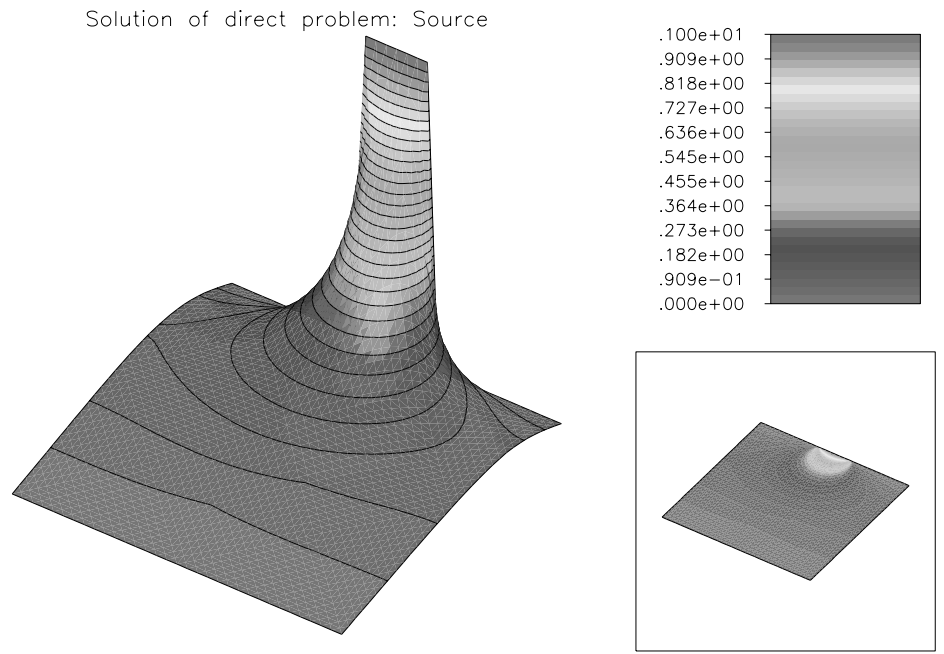

(b)

Figure 1 - Picture (a) show the doping profiles to be reconstructed in the numerical experiments.

object than the original unknown curve (the P-N junction). Due to the nature of the level set approach, it incorporates in a natural way the assumption that $\gamma$ is piecewise constant in $\Omega$. 

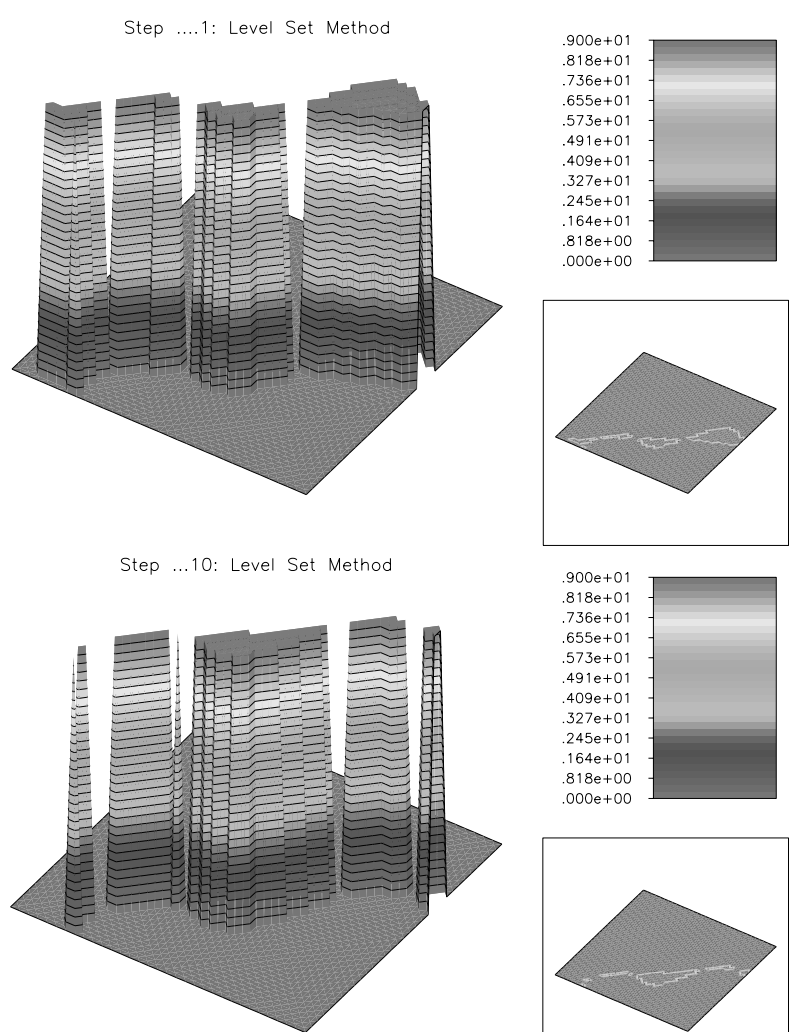

$.900 \mathrm{e}+01$ $.818 \mathrm{e}+01$ $.736 \mathrm{e}+01$ $.655 e+01$ $.573 \mathrm{e}+01$ $.491 \mathrm{e}+01$ $409 e+01$ $327 e+01$ $.245 \mathrm{e}+01$ $.164 \mathrm{e}+01$ $818 \mathrm{e}+00$ $.000 e+00$

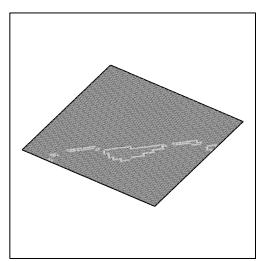

Step ..100: Level Set Method

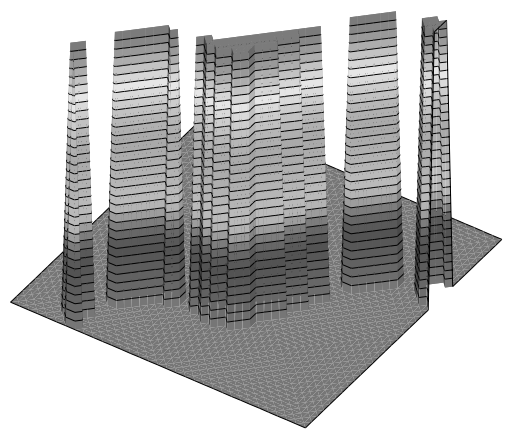

$.900 \mathrm{e}+01$ $.818 \mathrm{e}+01$ $.736 \mathrm{e}+01$ $573+01$ (5) $491 \mathrm{e}+01$ $.409 e+01$ $.327 e+01$ $.245 e+01$ $164 \mathrm{e}+01$ $818+00$ $818+00$



Figure 2 - Experiment for the bipolar model with pointwise measurements of the current density: Reconstruction of the p-n junction in Figure 1(a). Evolution of the iteration error for exact data and one measurement of the DN map $\Lambda_{\gamma}$ (i.e. $N=1$ in (13)). 


\section{Appendix}

Properties of silicon at room temperature

\begin{tabular}{cl}
\hline Parameter & \multicolumn{1}{c}{ Typical value } \\
\hline$\epsilon$ & $11.9 \epsilon_{0}$ \\
$\mu_{n}$ & $\approx 1500 \mathrm{~cm}^{2} \mathrm{~V}^{-1} \mathrm{~s}^{-1}$ \\
$\mu_{p}$ & $\approx 450 \mathrm{~cm}^{2} \mathrm{~V}^{-1} \mathrm{~s}^{-1}$ \\
$C_{n}$ & $2.8 \times 10^{-31} \mathrm{~cm}^{6} / \mathrm{s}$ \\
$C_{p}$ & $9.9 \times 10^{-32} \mathrm{~cm}^{6} / \mathrm{s}$ \\
$\tau_{n}$ & $10^{-6} \mathrm{~s}$ \\
$\tau_{p}$ & $10^{-5} \mathrm{~s}$ \\
\hline
\end{tabular}

Table 1 - Typical values of main the constants in the model.

Relevant physical constants:

Permittivity in vacuum: $\epsilon_{0}=8.85 \times 10^{-14} \mathrm{As} \mathrm{V}^{-1} \mathrm{~cm}^{-1}$;

Elementary charge: $q=1.6 \times 10^{-19}$ As.

Acknowledgments. The author was partially supported by the Brazilian National Research Council CNPq, grants 305823/03-5 and 478099/04-5.

\section{REFERENCES}

[1] K. Astala, L. Päivärinta and M. Lassas, Calderón's inverse problem for anisotropic conductivity in the plane. Comm. Partial Differential Equations, 30 (2005), 207-224.

[2] L. Borcea, Electrical impedance tomography. Inverse Problems, 18 (2002), R99-R136.

[3] A. Bukhgeim and G. Uhlmann, Recovering a potential from partial Cauchy data. Comm. Partial Differential Equations, 27 (2002), 653-668.

[4] M. Burger, H.W. Engl, A. Leitão and P.A. Markowich, On inverse problems for semiconductor equations. Milan Journal of Mathematics, 72 (2004), 273-314.

[5] M. Burger, H.W. Engl, P.A. Markowich and P. Pietra, Identification of doping profiles in semiconductor devices. Inverse Problems, 17 (2001), 1765-1795.

[6] M. Burger, H.W. Engl and P.A. Markowich, Inverse doping problems for semiconductor devices. In: Chan, T.F. et al (eds) Recent Progress in Computational and Applied PDEs. Kluwer/Plenum, New York, 39-53 (2002). 
[7] S. Busenberg and W. Fang, Identification of semiconductor contact resistivity. Quart. Appl. Math., 49 (1991), 639-649.

[8] S. Busenberg, W. Fang and K. Ito, Modeling and analysis of laser-beam-inducted current images in semiconductors. SIAM J. Appl. Math., 53 (1993), 187-204.

[9] H.W. Engl, M. Hanke and A. Neubauer, Regularization of Inverse Problems. Kluwer Academic Publishers, Dordrecht (1996).

[10] H.W. Engl, K. Kunisch and A. Neubauer, Convergence rates for Tikhonov regularization of nonlinear ill-posed problems. Inverse Problems, 5 (1989), 523-540.

[11] W. Fang and E. Cumberbatch, Inverse problems for metal oxide semiconductor field-effect transistor contact resistivity. SIAM J. Appl. Math., 52 (1992), 699-709.

[12] W. Fang and K. Ito, Identifiability of semiconductor defects from LBIC images. SIAM J. Appl. Math., 52 (1992), 1611-1626.

[13] W. Fang and K. Ito, Reconstruction of semiconductor doping profile from laser-beam-induced current image. SIAM J. Appl. Math., 54 (1994), 1067-1082.

[14] W. Fang, K. Ito and D.A. Redfern, Parameter identification for semiconductor diodes by LBIC imaging. SIAM J. Appl. Math., 62 (2002), 2149-2174.

[15] F. Frühauf, O. Scherzer and A. Leitão, Analysis of regularization methods for the solution of ill-posed problems involving discontinuous operators. SIAM J Numerical Analysis, 43 (2005), 767-786.

[16] H. Gajewski, On existence, uniqueness and asymptotic behavior of solutions of the basic equations for carrier transport in semicinductors. Z. Angew. Math. Mech., 65 (1985), 101108.

[17] P. Grisvard, Elliptic Problems in Nonsmooth Domains. Pittman Publishing, London, (1985).

[18] V. Isakov, Inverse problems for partial differential equations. Applied Mathematical Sciences, Springer, New York (1998).

[19] A. Leitão and O. Scherzer, On the relation between constraint regularization, level sets, and shape optimization. Inverse Problems, 19 (2003), L1-L11.

[20] A. Leitão, P.A. Markowich and J.P. Zubelli, On inverse doping profile problems for the voltage-current map. Inverse Problems, 22 (2006), 1071-1088.

[21] P.A. Markowich, The Stationary Semiconductor Device Equations. Springer, Vienna (1986).

[22] P.A. Markowich, C.A. Ringhofer and C. Schmeiser, Semiconductor Equations. Springer, Vienna (1990).

[23] A.I. Nachman, Global uniqueness for a two-dimensional inverse boundary value problem. Ann. of Math., 143 (1996), 71-96.

[24] S. Selberherr, Analysis and Simulation of Semiconductor Devices. Springer, New York (1984). 
[25] W.R. van Roosbroeck, Theory of flow of electrons and holes in germanium and other semiconductors. Bell Syst. Tech. J., 29 (1950), 560-607.

[26] A.N. Tikhonov and V.Y. Arsenin, Solutions of Ill-posed Problems. John Wiley \& Sons, New York (1977). 